International Journal of Pure and Applied Mathematics

Volume 84 No. $1 \quad$ 2013, 13-27

ISSN: 1311-8080 (printed version); ISSN: 1314-3395 (on-line version)

url: http://www.ijpam.eu

doi: http://dx.doi.org/10.12732/ijpam.v84i1.2

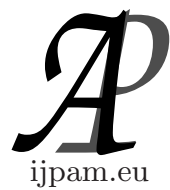

\title{
SOME SUZUKI TYPE FIXED POINT THEOREMS FOR GENERALIZED CONTRACTIVE MULTIFUNCTIONS
}

\author{
Ramendra Krishna Bose \\ Department of Mathemtics \\ University of Texas-PanAmerican \\ Edinburg, Texas 78539, USA
}

\begin{abstract}
The Banach contraction principle plays a very important role in nonlinear analysis and has many genralizations. Recently Suzuki (2008) gave a new generalization. Then, his method was extended by Kikkawa and Suzuki (2008). Then, Mot and Petrusel (2009), Dhompapangasa and Yingtaweesttikulue (2009), Bose and Roychowdhury (2011), Singh and Mishra (2010), and Doric and Lazovic (2011) further extended their work. In this paper, some results (Theorem 3.1 and Theorem 3.4 and their corollaries) in this direction concerning (common) fixed point theorems for generalized contractive multivalued mappings are presented using a result of Bose and Mukherjee(1977) which extend the results obtained by Bose and Mukherjee, Bose and Roychowdhury (with some constraint), Singh and Mishra, Kikkawa and Suzuki, Mot and Petrusel and others. In Theorem 3.7, a coincidence point theorem for a hybrid pair of mappings $f: X \rightarrow X$ and $T: X \rightarrow C B(X)$ is presented.
\end{abstract}

AMS Subject Classification: 47H10, 47H04, 54H25

Key Words: common fixed point, coincidence point, contractive multifunction, Hausdorff metric

Received: September 17, 2012

(c) 2013 Academic Publications, Ltd. url: www.acadpubl.eu 


\section{Introduction}

The Banach contraction principle plays a very impotant role in nonlinear analysis and has several generalizations([8] and the references there in). Recently, Suzuki [12] gave a new type of generalization of the Banach contraction principle. Then Kikkawa and Suzuki [8] gave another generalization which generalized the work of Suzuki and the Nadler fixed point theorem. Very Recently Bose and Roychowdhury [3] presented a theorem concerning $(\theta, L)$-multivalued weak contraction which generalized the work of Kikkawa and Suzuki, Nadler [10], Kamran [7], and Berinde and Berinde [1]. Also Mot and Petrusel [9] gave another generalization concerning special multivalued generalized contractions which extended the result of Kikkawa and Suzuki. Singh and Mishra[11] obtained a similar type theorem for a generalized contraction In this paper, some Kikkawa-Suzuku type theorems concerning the generalized multivalued contractions are presented which extends the work of Singh and Mishra [11], Bose and Roychowdhury [3], Mot and Petrusel [9], Kikkawa and Suzuki [8], and others. Also we prove a new coincidence point theorem concerning a hybrid pair of mappings $f: X \rightarrow X$ and $T: X \rightarrow C B(X)$.

\section{Peliminaries}

Let $(X, d)$ be a metric space and let $H$ represents the Hausdorff metric induced by the metric $d$. Let $C B(X)$ denotes the set of nonempty closed and bounded subsets of $X, K(X)$ be the set of nonempty compact subsets of $X$ and $C(X)$ be the family of nonempty closed subsets of $X$;

$$
H(A, B)=\max \{\rho(A, B), \rho(B, A)\} \text { where } \rho(A, B)=\sup _{a \in A} D(a, B),
$$

and $D(a, B)=\inf _{b \in B} d(a, b)$. Note that $(C B(X), H)$ is a complete metric space when $(X, d)$ is a complete metric space. Also $(C(X), H)$ is a complete generalized metric space( in the sense of Luxemburg-Jung[3]).

Theorem A. (Bose and Mukherjee [2]) Let $(X, d)$ be a complete metric space. Let $C B(X)$ denote the space of nonempty closed bounded subsets of $X$ equipped with the Hausdorff metric $H$. Let $F_{1}, F_{2}$ be mappings from $X$ into $C B(X)$ satisfying the following conditions:

$$
\begin{aligned}
H\left(F_{1} x, F_{2} y\right) \leq a_{1} D\left(x, F_{1} x\right)+a_{2} D & \left(y, F_{2} y\right) \\
& +a_{3} D\left(y, F_{1} x\right)+a_{4} D\left(x, F_{2} y\right)+a_{5} d(x, y),
\end{aligned}
$$


for all $x, y \in X$, where $a_{i}, i=1,2,3,4,5$ are non-negative numbers and $\sum_{0}^{5} a_{i}<$ 1 and $a_{1}=a_{2}$ or $a_{3}=a_{4}$.

Then there exists a point $x$ such that $x \in F_{1}(x)$ and $x \in F_{2}(x)$, i.e. $x$ is a common fixed point of $F_{1}$ and $F_{2}$.

When $F_{1}=F_{2}=F$ we have the following theorem:

Theorem B. (Bose and Mukherjee [2]) Let $(X, d)$ be a complete metric space. Let $C B(X)$ denote the space of nonempty closed bounded subsets of $X$ equipped with the Hausdorff metric $H$. Let $F$ be a mapping from $X$ into $C B(X)$ satisfying the following conditions:

$$
\begin{aligned}
H(F x, F y) \leq a_{1} D(x, F x)+a_{2} D(y, F y) & \\
& +a_{3} D(y, F x)+a_{4} D(x, F y)+a_{5} d(x, y)
\end{aligned}
$$

for all $x, y \in X$, where $a_{i}, i=1,2,3,4,5$ are non-negative numbers and $\sum_{0}^{5} a_{i}<$ 1 and $a_{1}=a_{2}$ and $a_{3}=a_{4}$.

Then there exists a point $x$ such that $x \in F(x)$, i.e., $x$ is a fixed point of $F$.

Theorem C. (Kikkawa and Suzuki [8]) Let $(X, d)$ be a complete metric space and $F: X \rightarrow C B(X)$ a multivalued mapping. Assume that there exists $r \in[0,1)$ such that $\frac{1}{1+r} D(x, F x) \leq d(x, y)$ implies that $H(F x, F y) \leq r d(x, y)$ for all $x, y \in X$. Then $F$ has a fixed pont.

Theorem D. (Suzuki [12]) For a metric space $(X, d), X$ is complete if and only if every mapping $T$ on $X$ such that there exists $r \in[0,1), \theta(r) D(x, T x) \leq$ $d(x, y)$ implies that $d(T x, T y) \leq r d(x, y$ for all $x, y \in X$, has a fixed point where $\theta:[0,1) \rightarrow\left(\frac{1}{2}, 1\right)$ is a onto and nonincreasing function defined below:

$$
\theta(r)=\left\{\begin{array}{l}
1,0 \leq r \leq \frac{\sqrt{5}-1}{2} \\
(1-r) r^{-2}, \frac{\sqrt{5}-1}{2} \leq r \leq 2^{-\frac{1}{2}} \\
(1+r)^{-1}, 2^{-\frac{1}{2}} \leq r<1
\end{array}\right.
$$

Later, Mot and Petrusel extended the Theorm $\mathrm{C}$ as follows:

Theorem E. (Mot and Petrusel [9]) Let $(X, d)$ be a complete metric space and $F: X \rightarrow C(X)$ a multifunction. Assume that there exist $\alpha . \beta, \gamma \in[0,1)$ such that $\alpha+\beta+\gamma<1$ and $\frac{1-\beta-\gamma}{1+\alpha} D(x, F x) \leq d(x, y)$ implies that $H(F x, F y) \leq$ $\alpha d(x, y)+\beta D(x, F x)+\gamma D(y, F y)$ for all $x, y \in X$. Then $F$ has a fixed point. 
Definition 2.1. Let $(X, d)$ be a metric space and let $F: X \rightarrow C(X) / C B(X)$ a muti-valued operator. $F$ is said to be a multi-valued weakly Picard operator (briefly MWP operator) if for all $x \in X$ and all $y \in F x$, there exists a sequence $\left\{x_{n}\right\}$ such that $x_{n+1} \in F x_{n}$, for all for all $n=0,1,2,3 \ldots$ starting with any $x_{0} \in X$ and the sequence is convergent and its limit is a fixed point of $F$.

Definition 2.2. (see [3]) Let $(X, d)$ be a metric space, $f: X \rightarrow X$ be a self-mapping and $T: X \rightarrow C B(X)$ be a multi-valued mapping. The mapping $f$ and $T$ are called $R$-weakly commuting if for given $x \in X, f(T(x)) \in C B(X)$ and there exists some real number $R$ such that

$$
H(f(T(x)), T(f(x))) \leq R d(f(x), T(x)) .
$$

Definition 2.3. (see [3]) The mappings $f: X \rightarrow X$ and $T: X \rightarrow C B(X)$ are weakly compatible if they commute at their coincidence poinyts, i.e. if $f(T(x))=T(F(x))$ whenever $f(x) \in T(x)$.

Theorem F. (Bose and Roychowdhury [3], Theorem 3.2) Let $(X, d)$ be a complete metric space and $T: X \rightarrow C B(X)$ a multivalued mapping and $f: X \rightarrow X$. Suppose that there exist two constants $\theta \in[0.1)$ and $L \geq 0$ such that

$$
\begin{aligned}
\eta(\theta) d(f(x), T x) \leq d(f(x), & f(y)) \\
& \Longrightarrow H(T x, T y \leq \theta d(f(x), f(y)))+L d(f(y), T x)
\end{aligned}
$$

for all $x, y \in X$, where $\eta:[0,1) \rightarrow\left(\frac{1}{2+L}, \frac{1}{1+L}\right)$ defined by $\eta(\theta)=\frac{1}{1+\theta+L}$ is a strictly decreasing function, $T(X) \subset f(X)$ and $f(X)$ is complete. Then:

(i) the set of coincidence point of $f$ and $T$ is non-empty, that is, there exists $u \in X$ such that $f(u) \in T u$.

(ii) for any $x_{0} \in X$, there exists an $f$-orbit $O_{f}\left(x_{0}\right)=\left\{f\left(x_{n}\right): n=1,2,3, \ldots\right\}$ of $T$ at the point $x_{0}$ such that $f\left(x_{n}\right) \rightarrow f(u)$, where $u$ is a coincidence point of $f$ and $T$, for which the following estimates hold:

$$
\begin{aligned}
& d\left(f\left(x_{n}\right), f(u)\right) \leq \frac{h^{n}}{1-h} d\left(f\left(x_{0}\right), f\left(x_{1}\right)\right), \quad n=0,1,2,3, \ldots \\
& d\left(f\left(x_{n}\right), f(u)\right) \leq \frac{h}{1-h} d\left(f\left(x_{n-1}\right), f\left(x_{n}\right)\right), \quad n=0,1,2,3, \ldots
\end{aligned}
$$

for a certain constant $h<1$. Further, if $f$ and $T$ are $R$-weakly commuting at $u$ and $f(f(u))=f(u)$, then $f$ and $T$ have a common fixed point. 
Lemma 2.4. (Nadler [10]) If $A, B \in C B(X)$ and $x \in A$, then for any positive number $\alpha$ there exists $y \in B$ such that $d(x, y) \leq H(A, B)+\alpha$, that is $d(x, y) \leq q H(A, B)$ where $q>1$.

\section{Main Theorems}

Here we present our main results.

Theorem 3.1. Let $(X, d)$ be a complete metric space. Let $C B(X)$ denote the space of nonempty closed bounded subsets of $X$ equipped with the Hausdorff metric $H$. Let $F$ and $G$ be a pair of mappings from $X$ into $C B(X)$ satisfying the following conditions:

$$
\begin{aligned}
& \alpha \min \{D(x, F x), D(y, G y)\} \leq d(x, y) \\
& \Longrightarrow H(F x, G y) \leq a_{1} D(x, F x)+a_{2} D(y, G y) \\
& \quad+a_{3} D(y, F x)+a_{4} D(x, G y)+a_{5} d(x, y)
\end{aligned}
$$

for all $x, y \in X$, where $a_{i}, i=1,2,3,4,5$ are non-negative numbers and $\sum_{0}^{5} a_{i}<$ 1 and $a_{1}=a_{2}$ or $a_{3}=a_{4}$. Here $\alpha=\min \{\gamma, \delta\}$ where $\gamma=\frac{1-a_{1}-a_{3}}{1-a_{3}+a_{4}+a_{5}}$ and $\delta=\frac{1-a_{2}-a_{4}}{1+a_{3}-a_{4}+a_{5}}$.

Then there exists a point $u \in X$ such that $u \in F(u)$ and $u \in G(u)$, i.e. $u$ is a common fixed point of $F$ and $G$.

Proof. Starting with arbitrary $x_{0} \in X$, and $x_{1} \in G x_{0}$, by Lemma 2.4, there exists $x_{2} \in F x_{1}$ such that $d\left(x_{1}, x_{2}\right) \leq p^{-1} H\left(F x_{1}, G x_{0}\right)$ where $p=\left(\sum_{0}^{5} a_{i}\right)^{\frac{1}{2}}$. This implies that

$$
\begin{aligned}
& p d\left(x_{1}, x_{2}\right) \leq a_{1} D\left(x_{1}, F x_{1}\right)+a_{2} D\left(x_{0}, G x_{0}\right)+a_{3} D\left(x_{0}, F x_{1}\right)+a_{4} D\left(x_{1}, G x_{0}\right) \\
& +a_{5} d\left(x_{1}, x_{0}\right) \leq a d\left(x_{1}, x_{2}\right)+a_{2} d\left(x_{0}, x_{1}\right)+a_{3} d\left(x_{0}, x_{2}\right)+a_{4} .0+a_{5} d\left(x_{1}, x_{0}\right) .
\end{aligned}
$$

This leads to $d\left(x_{1}, x_{2}\right) \leq \frac{a_{2}+a_{3}+a_{5}}{p-a_{1}-a_{3}} d\left(x_{1}, x_{0}\right)=r d\left(x_{1}, x_{0}\right)$. Similarly, by Lemma 2.4, there exists $x_{3} \in G x_{2}$ such that

$$
d\left(x_{2}, x_{3}\right) \leq \frac{a_{1}+a_{4}+a_{5}}{p-a_{2}-a_{4}} d\left(x_{1}, x_{2}\right)=\operatorname{sd}\left(x_{1}, x_{2}\right) \leq \operatorname{srd}\left(x_{1}, x_{0}\right) .
$$

More specifically, define $x_{2 n+1} \in G x_{2 n}$ and $x_{2 n+2} \in F x_{2 n+1}$ starting with $x_{0} \in X$ (That is, starting with $x_{1} \in G\left(x_{0}\right)$ and $x_{2} \in F\left(x_{1}\right)$ and following Bose and Mukherjee [2]). Then we show that

$$
\alpha\left\{D\left(x_{2 n-1}, F x_{2 n-1}\right), D\left(x_{2 n}, G x_{2 n}\right)\right\} \leq d\left(x_{2 n-1}, x_{2 n}\right) .
$$


Case I. $D\left(x_{2 n-1}, F x_{2 n-1}\right) \geq D\left(x_{2 n}, G x_{2 n}\right)$ :

Since

$$
D\left(x_{2 n}, G x_{2 n}\right) \leq D\left(x_{2 n-1}, F x_{2 n-1}\right) \leq d\left(x_{2 n-1}, x_{2 n}\right)
$$

we have

$$
\alpha\left\{D\left(x_{2 n-1}, F x_{2 n-1}\right), D\left(x_{2 n}, G x_{2 n}\right)\right\} \leq d\left(x_{2 n-1}, x_{2 n}\right)
$$

Case II. $D\left(x_{2 n-1}, F x_{2 n-1}\right) \leq D\left(x_{2 n}, G x_{2 n}\right)$ :

Since $D\left(x_{2 n-1}, F x_{2 n-1}\right) \leq d\left(x_{2 n-1}, x_{2 n}\right)$ we have the desired inequality since $\alpha \in(0,1)$. This implies

$$
\begin{aligned}
& d\left(x_{2 n}, x_{2 n+1}\right) \leq p^{-1} H\left(F x_{2 n-1}, G x_{2 n}\right) \leq(r s)^{n} d\left(x_{0}, x_{1}\right), \quad \text { and } \\
& d\left(x_{2 n+1}, x_{2 n+2}\right) \leq p^{-1} H\left(F x_{2 n+1}, G x_{2 n}\right) \leq(r s)^{n} r d\left(x_{0}, x_{1}\right)
\end{aligned}
$$

where $p=\left(\sum_{0}^{5} a_{i}\right)^{\frac{1}{2}}$ and $r=\frac{a_{2}+a_{3}+a_{5}}{p-a_{1}-a_{3}}$ and $s=\frac{a_{1}+a_{4}+a_{5}}{p-a_{2}-a_{4}}$. We have $0<r, s<1$ if $a_{3}=a_{4}$ and $0<r s<1$ when $a_{1}=a_{2}$ or $a_{3}=a_{4}$. Further,

$$
\sum_{n=0}^{\infty}\left[d\left(x_{2 n}, x_{2 n+1}\right)+d\left(x_{2 n+1}, x_{2 n+2}\right)\right] \leq(1+r) d\left(x_{0}, x_{1}\right) \sum_{n=0}^{\infty}(r s)^{n}
$$

From this, it is easily seen that the sequence $\left\{x_{n}\right\}$ is Cauchy and the sequence converges to some point $u \in X$.

Next we prove the following: (a) $\gamma D(u, F x) \leq d(u, x)$ (b) $\delta d(u, G x) \leq$ $d(u, x)$ for all $x \in X-\{u\}$.

Since $x_{n} \rightarrow u$, there exists $n_{0} \in N$ such that $d\left(u, x_{n}\right) \leq \frac{1}{3} d(u, y)$ for $y \neq u$ and all $n \geq n_{0}$. We have

$$
\begin{aligned}
\alpha D\left(x_{2 n-1}, F x_{2 n-1}\right) & <D\left(x_{2 n-1}, F x_{2 n-1}\right) \leq d\left(x_{2 n-1}, x_{2 n}\right) \\
& \leq d\left(x_{2 n-1}, u\right)+d\left(u, x_{2 n}\right) \leq \frac{2}{3} d(y, u) \\
& =d(y, u)-\frac{1}{3} d(y, u) \leq d(y, u)-d\left(x_{2 n-1}, u\right) \leq d\left(x_{2 n-1}, y\right)
\end{aligned}
$$

Therefore $\alpha D\left(x_{2 n-1}, F x_{2 n-1}\right)<d\left(x_{2 n-1}, y\right)$. Now either $D\left(x_{2 n-1}, F x_{2 n-1}\right) \leq$ $D(y, G y)$ or $D\left(x_{2 n-1}, F x_{2 n-1}\right) \geq D(y, G y)$. In either case, we have $\alpha \min \left\{D\left(x_{2 n-1}, F x_{2 n-1}\right), D(y, G y) \leq d\left(x_{2 n-1}, y\right)\right\}$. Hence

$$
D\left(x_{2 n}, G y\right) \leq H\left(F x_{2 n-1}, G y\right) \leq a_{1} D\left(x_{2 n-1}, F x_{2 n-1}\right)+a_{2} D(y, G y)
$$




$$
\begin{aligned}
& +a_{3} D\left(y, F x_{2 n-1}\right)+a_{4} D\left(x_{2 n-1}, G y\right)+a_{5} d\left(x_{2 n-1}, y\right) \\
\leq & a_{1} d\left(x_{2 n-1}, x_{2 n}\right) \\
& +a_{2} D(y, G y)+a_{3} D\left(y, x_{2 n}\right)+a_{4} D\left(x_{2 n-1}, G y\right)+a_{5} d\left(x_{2 n-1}, y\right)
\end{aligned}
$$

Taking limit as $n \rightarrow \infty$, we have

$$
D(u, G y) \leq a_{2} D(y, G y)+a_{3} D(y, u)+a_{4} D(u, G y)+a_{5} d(u, y)
$$

and this leads to $D(u, G y) \leq \frac{a_{2}+a_{3}+a_{5}}{1-a_{2}-a_{4}} d(u, y)$ and similarly we can show that $D(u, F y) \leq \frac{a_{1}+a_{4}+a_{5}}{1-a_{1}-a_{3}} d(u, y)$.

When $x \neq u$, for every $n \in N$ ther exists $z_{n} \in F x$ such that $d\left(u, z_{n}\right) \leq$ $D(u, F x)+\frac{1}{n} d(x, u)$. Also we have

$$
\begin{aligned}
D(x, F x) \leq d\left(x, z_{n}\right) \leq d(x, u)+d\left(u, z_{n}\right) & \leq d(x, u) \\
+D(u, F x)+\frac{1}{n} d(x, u) & \leq\left[1+\frac{a_{1}+a_{4}+a_{5}}{1-a_{1}-a_{3}}+\frac{1}{n}\right] d(u, x) .
\end{aligned}
$$

Taking limit as $n \rightarrow \infty$, we have

$$
D(x, F x) \leq\left[\frac{1-a_{3}+a_{4}+a_{5}}{1-a_{1}-a_{3}}\right] d(u, x) .
$$

That is, $\frac{1-a_{1}-a_{3}}{1-a_{3}+a_{4}+a_{5}} D(x, F x) \leq d(u, x)$ and similarly we have

$$
\frac{1-a_{4}+a_{3}+a_{5}}{1-a_{2}-a_{4}} d(x, G x) \leq d(u, x) .
$$

Let $\gamma=\frac{1-a_{1}-a_{3}}{1-a_{3}+a_{4}+a_{5}}$ and $\delta=\frac{1-a_{2}-a_{4}}{1-a_{4}+a_{3}+a_{5}}$. Let $\alpha=\min \{\gamma, \delta\}$.

We have by similar argument, $\alpha \min \{D(x, F x), D(u, G u)\} \leq d(u, x)$. This implies that

$$
\begin{aligned}
H(F x, G u) \leq a_{1} D(x, F x)+a_{2} D(u, G u)+a_{3} D u y & , F x) \\
& +a_{4} D(x, G u)+a_{5} d(x, u) .
\end{aligned}
$$

Taking $x=x_{2 n+2}$ we have

$$
\begin{aligned}
& D\left(x_{2 n+2}, G u\right) \leq H\left(F x_{2 n+1}, G u\right) \leq a_{1} D\left(x_{2 n+1}, F x_{2 n+1}\right) \\
& \quad+a_{2} D(u, G u)+a_{3} D\left(u, F x_{2 n+1}\right)+a_{4} D\left({ }_{2 n+1} x, G u\right)+a_{5} d\left(x_{2 n+1}, u\right)
\end{aligned}
$$

Taking limit as $n \rightarrow \infty$, we have $D(u, G u) \leq a_{1} .0+a_{2} D(u, G u)+a_{3} .0+$ $a_{4} D(u, G u)+a_{5} .0$. That is $\left(1-a_{2}-a_{4}\right) D(u, G u) \leq 0$. This implies $D(u, G u)=$ 
0 since $1-a_{2}-a_{4}>0$. That is $u \in G u$. Similarly we can show that $u \in F u$. That is, $u \in F u \cap G u$.

If $F=G$, then adopting new notations, $a_{1}=a_{2}=a, \quad a_{3}=a_{4}=b$ and $a_{5}=c$, we have the following Corollary:

Corollary 3.2. Let $(X, d)$ be a complete metric space. Let $C B(X)$ denote the space of nonempty closed bounded subsets of $X$ equipped with the Hausdorff metric $H$. Let $F$ be a mapping from $X$ into $C B(X)$ satisfying the following conditions:

For each $x, y \in X$ we have $\alpha D(x, F x) \leq d(x, y)$ implies

$$
H(F x, F y) \leq a[D(x, F x)+D(y, F y)]+b[D(y, F x)+D(x, F y)]+c d(x, y),
$$

where $a, b, c$ are non-negative real numbers and $2 a+2 b+c<1$. Then there exists a point $x \in X$ such that $x \in F(x)$, i.e. Fix $(F) \neq \phi$. Moreover, $F$ is a $M W F$ operator. Here $\alpha=\frac{1-a-b}{1+c}$ (nontrivial case when $\alpha=\frac{1-a-b}{1+c}<1$ ).

If in the above corollary, $F: X \rightarrow X$, then we have the following corollary:

Corollary 3.3. Let $(X, d)$ be a complete metric space. Let $F$ and $G$ be a pair of mappings from $X$ into into itself satisfying the following conditions:

$$
\begin{aligned}
& \alpha \min \{d(x, F x), d(y, G y)\} \leq d(x, y) \\
& \Longrightarrow d(F x, G y) \leq a_{1} d(x, F x)+a_{2} d(y, G y) \\
& \quad+a_{3} d(y, F x)+a_{4} d(x, G y)+a_{5} d(x, y)
\end{aligned}
$$

for all $x, y \in X$, where $a_{i}, i=1,2,3,4,5$ are non-negative numbers and $\sum_{0}^{5} a_{i}<$ 1 and $a_{1}=a_{2}$ or $a_{3}=a_{4}$. Here $\alpha=\min \{\gamma, \delta\}$ where

$$
\gamma=\frac{1-a_{1}-a_{3}}{1-a_{3}+a_{4}+a_{5}} \quad \text { and } \quad \delta=\frac{1-a_{2}-a_{4}}{1+a_{3}-a_{4}+a_{5}}
$$

Then there exists a point $u \in X$ such that $u=F(u)$ and $u=G(u)$, i.e. $u$ is a common fixed point of $F$ and $G$.

Corollary 3.4. Let $(X, d)$ be a complete metric space and let $F: X \rightarrow X$ which satidfies the following conditions:

For each $x, y \in X$ we have $\alpha d(x, F x) \leq d(x, y)$ implies

$$
d(F x, F y) \leq a[d(x, F x)+d(y, F y)]+b[d(y, F x)+d(x, F y)]+c d(x, y),
$$

where $a, b, c$ are non-negative real numbers and $2 a+2 b+c<1$. Then there exists a unique point $x \in X$ such that $x=F(x)$. Moreover, $F$ is a $M W F$ operator. Here $\alpha=\frac{1-a-b}{1+c}$ (nontrivial case when $\alpha=\frac{1-a-b}{1+c}<1$ ). 
Remark 3.5. Corollary 3.2 is same as Corollary 4.4 of [11] given in the setting of the space $(C(X), H)$. However when we try to get the Theorem 6.6 of Mot and Petrusel [9] (that is, of Theorem D) from this, putting $c=0$, we get $\alpha=\frac{1-b}{1+c}$ whereas $\alpha=\frac{1-2 b}{1+c}$ in Mot and Petrusel. Hence we present an independent proof of Corollary 3.2, following the approach of Mot and Petrusel, with a different $\alpha$ in the next theorem and we can recover Theorem 6.6 of Mot and Petrusel as a particular case from this theorem.

Theorem 3.6. Let $(X, d)$ be a complete metric space. Let $C B(X)$ denote the space of nonempty closed bounded subsets of $X$ equipped with the Hausdorff metric $H$. Let $F$ be a mapping from $X$ into $C B(X)$ satisfying the following conditions:

For each $x, y \in X$ we have $\frac{1-2 a-b}{1+c+b} D(x, F x) \leq d(x, y)$ implies

$$
H(F x, F y) \leq a[D(x, F x)+D(y, F y)]+b[D(y, F x)+D(x, F y)]+c d(x, y),
$$

where $a, b, c$ are non-negative real numbers and $2 a+2 b+c<1$. Then there exists a point $x \in X$ such that $x \in F(x)$, i.e. $F i x(F) \neq \phi$. Moreover, $F$ is a $M W F$ operator.

Proof. Let $x_{0} \in X$. Choose $x_{1} \in F x_{0}$. Since $\eta D\left(x_{0}, F x_{0}\right) \leq \eta d\left(x_{0}, x_{1}\right) \leq$ $d\left(x_{0}, x_{1}\right)$ where $\eta=\frac{1-2 a-b}{1+c+b} \leq 1$ (nontrivial case when $\eta=\frac{1-2 a-b}{1+c+b}<1$ ). This implies

$$
\begin{aligned}
H\left(F x_{0}, F x_{1}\right) \leq a\left[D\left(x_{0}, F x_{0}\right)+\right. & \left.D\left(x_{1}, F x_{1}\right)\right] \\
& +b\left[D\left(x_{0}, F x_{1}\right)+D\left(x_{1}, F x_{0}\right)\right]+c d\left(x_{0}, x_{1}\right) .
\end{aligned}
$$

By Lemma 2.4, there exists $x_{2} \in F\left(x_{1}\right)$ such that $d\left(x_{1}, x_{2}\right) \leq p^{-1} H\left(F x_{0}, F x_{1}\right)$ where $p=(2 a+2 b+c)^{\frac{1}{2}}, p^{-1}>1$.

We have

$$
\begin{aligned}
& d\left(x_{1}, x_{2}\right) \leq p^{-1} H\left(F x_{0}, F x_{1}\right) \\
& \leq p^{-1}\left[a\left[D\left(x_{0}, F x_{0}\right)+D\left(x_{1}, F x_{1}\right)\right]+b\left[D\left(x_{0}, F x_{1}\right)+D\left(x_{1}, F x_{0}\right)\right]+c d\left(x_{0}, x_{1}\right)\right] \\
& \leq p^{-1}\left[a\left[d\left(x_{0}, x_{1}\right)+d\left(x_{1}, x_{2}\right)\right]+b\left[d\left(x_{0}, x_{2}\right)+0\right]+c d\left(x_{0}, x_{1}\right)\right] \\
& \Rightarrow p d\left(x_{1}, x_{2}\right)-a d\left(x_{1}, x_{2}\right)-b d\left(x_{1}, x_{2}\right) \leq a d\left(x_{0}, x_{1}\right)+b d\left(x_{0}, x_{1}\right)+c d\left(x_{0}, x_{1}\right) \\
& \Rightarrow d\left(x_{1}, x_{2}\right) \leq \frac{a+b+c}{p-a-b} d\left(x_{0}, x_{1}\right)=r d\left(x_{0}, x_{1}\right)
\end{aligned}
$$

Since $\eta D\left(x_{1}, F x_{1}\right) \leq \eta d\left(x_{1}, x_{2}\right) \leq d\left(x_{1}, x_{2}\right)$ This implies 


$$
\begin{aligned}
H\left(F x_{1}, F x_{2}\right) \leq a\left[D\left(x_{1}, F x_{1}\right)+\right. & \left.D\left(x_{2}, F x_{2}\right)\right] \\
& +b\left[D\left(x_{1}, F x_{2}\right)+D\left(x_{2}, F x_{1}\right)\right]+c d\left(x_{1}, x_{2}\right) .
\end{aligned}
$$

By Lemma 2.4, there exists $x_{3} \in F x_{2}$ such that $d\left(x_{2}, x_{3}\right) \leq p^{-1} H\left(F x_{1}, F x_{2}\right)$. This leads similarly to $d\left(x_{2}, x_{3}\right) \leq \frac{a+b+c}{p-a-b} d\left(x_{1}, x_{2}\right)=r^{2} d\left(x_{0}, x_{1}\right)$, where $0<r<$ 1. Similarly we generate a sequence $\left\{x_{n}\right\}$ starting from $x_{0}$ such that $x_{n+1} \in F x_{n}$ for each $n \in N$ and $d\left(x_{n}, x_{n+1}\right) \leq r^{n} d\left(x_{0}, x_{1}\right)$ for each $n \in N$. Thus $\left\{x_{n}\right\}$ is a Cauchy sequence in $(X, d)$. Let $z$ be the limit of this sequence.

Now we show that $D(z, F x) \leq\left(\frac{a}{\eta}+b+c\right) d(x, z)+a D(x, F x)+b D(z, F x)$, for each $x \in X \backslash\{z\}$. Suppose $x \neq z$. Since the sequence $\left\{x_{n}\right\}$ converges to $z$, there exists $n_{0} \in N$ such that $d\left(x_{n}, z\right) \leq \frac{1}{3} d(x, z)$ for each $n \geq n_{0}$. Then we have

$$
\begin{aligned}
\eta D\left(x_{n}, F x_{n}\right) & \leq D\left(x_{n}, F x_{n}\right) \leq d\left(x_{n}, x_{n+1}\right) \leq d\left(x_{n}, z\right)+d\left(z, x_{n+1}\right) \\
\leq & \frac{2}{3} d(x, z) \leq d(x, z)-\frac{1}{3} d(x, z) \leq d(x, z)-d\left(x_{n}, z\right) \leq d\left(x_{n}, x\right),
\end{aligned}
$$

that is, $\eta D\left(x_{n}, F x_{n}\right) \leq d\left(x_{n}, x\right), n \geq n_{0}$.

Hence

$$
\begin{aligned}
H\left(F x_{n}, F x\right) \leq & a\left[D\left(x_{n}, F x_{n}\right)+D(x, F x)\right]+b\left[D\left(x_{n}, F x\right)+D\left(x, F x_{n}\right)\right] \\
& +c d\left(x_{n}, x\right) \leq\left[\frac{a}{\eta}+c\right] d\left(x_{n}, x\right)+b d\left(x, x_{n+1}\right) \\
& +a D(x, F x)+b D\left(x_{n}, F x\right)
\end{aligned}
$$

since $x_{n+1} \in F x_{n}, n \geq n_{0}$. Now

$$
\begin{aligned}
D\left(x_{n+1}, F x\right) \leq H\left(F x_{n}, F x\right) \leq[ & \left.\frac{a}{\eta}+c\right] d\left(x_{n}, x\right) \\
& +b d\left(x, x_{n+1}\right)+a D(x, F x)+b D\left(x_{n}, F x\right) .
\end{aligned}
$$

Taking $n \rightarrow \infty$, we have

$$
(1-b) D(z, F x) \leq\left[\frac{a}{\eta}+c+b\right] d(x, z)+a D(x, F x) .
$$

Now we show that

$$
H(F x, F z) \leq\left[\frac{a}{\eta}+c\right] d(x, z)+a D(z, F z)+b D(x, F z)+b D(z, F x)
$$

for all $x \in X$. If $x=z$, it is satisfied. If $x \neq z$, there exists $y_{n} \in F x$ such that

$$
d\left(z, y_{n}\right) \leq D(z, F x)+\frac{1}{n} d(x, z), D(x, F x) \leq d\left(x, y_{n}\right) \leq d(x, z)+d\left(z, y_{n}\right)
$$




$$
\begin{aligned}
& \leq d(x, z)+D(z, F x)+\frac{1}{n} d(x, z) \\
& \leq d(x, z)+\left[\frac{\frac{a}{\eta}+c+b}{1-b} d(x, z)+\frac{a}{1-b} D(x, F x)\right]+\frac{1}{n} d(x, z) .
\end{aligned}
$$

This leads to

$$
\eta D(x, F x) \leq\left[\frac{\eta(1+b+c)+a}{1-a-b}+\frac{1}{n} \frac{1-a-b}{1-b}\right] d(x, z) .
$$

Hence as $n \rightarrow \infty$, we have

$$
\eta D(x, F x) \leq\left[\frac{\eta(1+b+c)+a}{1-a-b}\right] d(x, z)=d(x, z),
$$

since $\frac{\eta(1+b+c)+a}{1-a-b}=1$, that is, $\eta D(x, F x) \leq d(x, z)$. Hence

$$
\begin{aligned}
H(F x, F z) \leq & a[D(x, F x)+D(z, F x)]+b[D(x, F z)+D(z, F x)] \\
& +c d(x, z) \leq\left[\frac{a}{\eta}+c\right] d(x, z)+a D(z, F z)+b D(x, F z)+b D(z, F x) .
\end{aligned}
$$

Now

$$
\begin{aligned}
D(z, F z) & =\lim _{n \rightarrow \infty} D\left(x_{n+1}, F z\right) \leq \lim _{n \rightarrow \infty} H\left(F x_{n}, F z\right) \\
\leq & \lim _{n \rightarrow \infty}\left[\left[\frac{a}{\eta}+c\right] d\left(x_{n}, z\right)+a D(z, F z)+b D\left(x_{n}, F z\right)+b D\left(z, F x_{n}\right)\right] .
\end{aligned}
$$

Taking the limit we have $D(z, F x) \leq a D(z, F z)+b D(z, F z)$. That is, $(1-a-b) D(z, F z)=0 \Rightarrow D(z, F z)=0 \Rightarrow z \in F z$.

Corollary 3.7. If $b=0$ in Theorem 3.6, we get Theorem D.

Corollary 3.8. If $a=0$ and $b=0$ in Theorem 3.6 or Corollary 3.2 then we get Theorem $C$.

Following Theorem 3.2 of Bose and Roychowdhury [3] we can prove the following theorem and some other related results in similar fashion discussed there.

Theorem 3.9. Let $(X, d)$ be a metric space, $T: X \rightarrow C B(X)$ and $f$ : $X \rightarrow X$. Suppose for every $x, y \in X, \alpha D(f(x), T x) \leq d(f(x), f(y))$ implies

$$
H(T x, T y) \leq a[D(f(x), T x)+D(f(y), T y)]
$$




$$
+b[D(f(y), T x)+D(f(x), T y)]+c d(f(x), f(y)),
$$

where $\alpha=\frac{1-a-b}{1+c}(\leq 1)$. Also $T(X) \subset f(X)$ and $f(X)$ is complete. Then the set of coincidence point of $f$ and $T$ is non-empty, that is, there exists $u \in X$ such that $f(u) \in T u$.

(ii) for any $x_{0} \in X$, there exists an $f$-orbit $O_{f}\left(x_{0}\right)=\left\{f\left(x_{n}\right): n=1,2,3, \ldots\right\}$ of $T$ at the point $x_{0}$ such that $f\left(x_{n}\right) \rightarrow f(u)$, where $u$ is a coincidence point of $f$ and $T$, for which the following estimates hold:

$$
\begin{aligned}
& d\left(f\left(x_{n}\right), f(u)\right) \leq \frac{h^{n}}{1-h} d\left(f\left(x_{0}\right), f\left(x_{1}\right)\right), \quad n=0,1,2,3, \ldots \\
& d\left(f\left(x_{n}\right), f(u)\right) \leq \frac{h}{1-h} d\left(f\left(x_{n-1}\right), f\left(x_{n}\right)\right), \quad n=0,1,2,3, \ldots
\end{aligned}
$$

for a certain constant $h<1$. Further, if $f$ and $T$ are $R$-weakly commuting at $u$ and $f(f(u))=f(u)$, then $f$ and $T$ have a common fixed point.

Proof. Let $x_{0} \in X$ and $x_{1} \in X$ such that $f\left(x_{1}\right) \in T\left(x_{0}\right)$. Then

$$
\begin{aligned}
& \alpha D\left(f\left(x_{0}\right), T\left(x_{0}\right)\right) \leq d\left(f\left(x_{0}\right), f\left(x_{1}\right)\right) \Longrightarrow \\
& \quad H\left(T\left(x_{0}\right), T\left(x_{1}\right)\right) \leq a\left[D\left(f\left(x_{0}\right), T x_{0}\right)+D\left(f\left(x_{1}\right), T x_{1}\right)\right] \\
& \quad+b\left[D\left(f\left(x_{1}\right), T x_{0}\right)+D\left(f\left(x_{0}\right), T x_{1}\right)\right]+c d\left(f\left(x_{0}\right), f\left(x_{1}\right)\right) .
\end{aligned}
$$

Let $x_{2} \in X$ such that $f\left(x_{2}\right) \in T x_{1}$. Then

$$
d\left(f\left(x_{1}\right), f\left(x_{2}\right)\right) \leq p^{-1} H\left(T x_{0}, T x_{1}\right),
$$

where $p=(2 a+2 b+c)^{\frac{1}{2}}$. Thus we have

$$
\begin{aligned}
p d\left(f\left(x_{1}\right), f\left(x_{2}\right)\right) \leq a\left[D \left(f\left(x_{0}\right)\right.\right. & \left., T f\left(x_{0)}\right)+D\left(f\left(x_{1}\right), f\left(x_{2}\right)\right)\right] \\
& +b\left[0+D\left(f\left(x_{0}\right), T f\left(x_{2}\right)\right)\right]+c d\left(f\left(x_{0}\right), f\left(x_{1}\right)\right) .
\end{aligned}
$$

This leads to $d\left(f\left(x_{1}\right), f\left(x_{2}\right)\right) \leq \frac{a+b+c}{p-a-b} d\left(f\left(x_{0}\right), f\left(x_{1}\right)\right)=k d\left(f\left(x_{0}\right), f\left(x_{1}\right)\right)$ where $k=\frac{a+b+c}{p-a-b} \leq 1$. Let $x_{2} \in X$ such that $f\left(x_{2}\right) \in T x_{1}$. Then

$$
\begin{aligned}
& \alpha D\left(f\left(x_{1}\right), T\left(x_{1}\right)\right) \leq d\left(f\left(x_{1}\right), f\left(x_{2}\right)\right) \\
& \Longrightarrow H\left(T\left(x_{1}\right), T\left(x_{2}\right)\right) \leq a\left[D\left(f\left(x_{1}\right), T x_{1}\right)+D\left(f\left(x_{2}\right), T x_{2}\right)\right] \\
& \quad+b\left[D\left(f\left(x_{2}\right), T x_{1}\right)+D\left(f\left(x_{1}\right), T x_{2}\right)\right]+c d\left(f\left(x_{1}\right), f\left(x_{2}\right)\right) \\
& \Longrightarrow d\left(f\left(x_{2}\right), f\left(x_{3}\right)\right) \leq \frac{a+b+c}{p-a-b} d\left(f\left(x_{1}\right), f\left(x_{2}\right)\right)
\end{aligned}
$$




$$
=k d\left(f\left(x_{1}\right), f\left(x_{2}\right)\right) \leq k^{2} d\left(f\left(x_{0}\right), f\left(x_{1}\right)\right)
$$

It can easily be shown that the sequence $\left\{f\left(x_{n}\right)\right\}$ is a Cauchy sequence in $f(X)$. Since $f(X)$ is co mplete, the sequence converges to some point $f(u) \in f(X)$. So there exists a positive integer $\nu$ such that for all $x \in X \backslash\{u\}$, we have $d\left(f\left(x_{0}\right), f\left(x_{1}\right)\right) \leq \frac{1}{3} d(f(x), f(u))$ for $n \geq \nu$. Then, we can show that

$$
\begin{aligned}
& \alpha D\left(f\left(x_{n}\right), T\left(x_{n}\right)\right) \leq D\left(f\left(x_{n}\right), T\left(x_{n}\right)\right) \leq d\left(f\left(x_{n}\right), f\left(x_{n+1}\right)\right) \leq d\left(f\left(x_{n}\right), f(x)\right) \\
& \left.\Longrightarrow H\left(T\left(x_{n}\right), T(x)\right) \leq a\left[D\left(f\left(x_{n}\right), T x_{n}\right)+D(f(x), T x)\right)\right] \\
& +b\left[D\left(f(x), T x_{n}\right)+D\left(f\left(x_{n}\right), T x\right)\right]+c d\left(f\left(x_{n}\right), f(x)\right) \\
& \left.\Longrightarrow D\left(f\left(x_{n+1}\right), T x\right) \leq H\left(T\left(x_{n}\right), T(x)\right) \leq a\left[D\left(f\left(x_{n}\right), T x_{n}\right)+D(f(x), T x)\right)\right] \\
& +b\left[D\left(f(x), T x_{n}\right)+D\left(f\left(x_{n}\right), T x\right)\right]+c d\left(f\left(x_{n}\right), f(x)\right) \\
& \left.\leq a\left[D\left(f\left(x_{n}\right), f\left(x_{n+1}\right)\right)+D(f(x), T x)\right)\right] \\
& +b\left[D\left(f(x), f\left(x_{n+1}\right)\right)+D\left(f\left(x_{n}\right), T x\right)\right]+c d\left(f\left(x_{n}\right), f(x)\right)
\end{aligned}
$$

Taking limit as $n \rightarrow \infty$, we have

$$
\begin{aligned}
D(f(u), T x) \leq & a D(f(x), T x))+b[D(f(x), f(u))+D(f(u), T x)] \\
& +c d(f(x), f(u)) \Rightarrow D(f(u), T x) \leq \frac{a+b+c}{1-a-b} d(f(x), f(u)) .
\end{aligned}
$$

for all $x \in X \backslash\{u\}$.

Next we show that

$$
\begin{aligned}
& H(T x, T u) \leq a[D(f(x), T x))+D(f(u), T u)] \\
& +b[D(f(u), T x)+D(f(x), T u)]+c d(f(x), f(u))
\end{aligned}
$$

It is true if $x=u$. Suppose $x \neq u$, then for each $n \in \mathrm{N}$, we can obtain a sequence $\left\{v_{n}\right\}$ in $T x$ such that $d\left(f(u), v_{n}\right) \leq D(f(u), T x)+\frac{1}{n} d(f(x), f(u))$. Hence for

$$
\begin{aligned}
D(f(x), T x) & \leq d\left(f(x), v_{n}\right) \leq d(f(x), f(u))+d\left(f(u), v_{n}\right) \\
& \leq d(f(x), f(u))+D(f(u), T x)+\frac{1}{n} d(f(x), f(u)) \\
& \leq d(f(x), f(u))+\frac{a+b+c}{p-a-b} d(f(x), f(u))+\frac{1}{n} d(f(x), f(u)) \\
& =\left[\frac{1+c}{1-a-b}+\frac{1}{n}\right] d(f(x), f(u))
\end{aligned}
$$

for every $n$. That is, $\frac{1-a-b}{1+c} D(f(x), T x) \leq d(f(x), f(u))$. This implies that 


$$
\begin{aligned}
& H(T x, T u) \leq a[D(f(x), T x))+D(f(u), T u)] \\
& +b[D(f(u), T x)+D(f(x), T u)]+c d(f(x), f(u)) .
\end{aligned}
$$

Now

$$
\begin{aligned}
D(f(u), T u)= & \lim _{n \rightarrow \infty} D\left(f\left(x_{n+1}\right), T u\right) \leq H\left(T x_{n}, T u\right) \\
\leq & \lim _{n \rightarrow \infty}\left\{a\left[D\left(f\left(x_{n}\right), T x_{n}\right)\right)+D(f(u), T u)\right] \\
& \left.+b\left[D\left(f(u), T x_{n}\right)+D\left(f\left(x_{n}\right), T u\right)\right]+c d\left(f\left(x_{n}\right), f(u)\right)\right\} \\
\Longrightarrow & D(f(u), T u) \leq a D(f(u), T u)+b D(f(u), T u) .
\end{aligned}
$$

That is, we have $(1-a-b) D(f(u), T u) \leq 0 \Longrightarrow D(f(u), T u)=0$ and this implies that $f(u) \in T u$. The part (ii), especially the estimates can be proved similarly as in [3]. If $f$ and $T$ are weakly commuting at $u$, we have

$$
H(f(T(u)), T(f(u))) \leq R d(f(u), T(u)) .
$$

As $f(u) \in T u$, this implies $f(T(u))=T(f(u))$. Again $f(f(u))=f(u)$, and so $f(u) \in T u$ implies $f(f(u)) \in f(T(u))=T(f(u))$, i.e. $f(u) \in T(f(u))$. Hence, $f(u)$ is a fixed point of both $f$ and $T$, i.e., $f$ and $T$ have a common fixed point.

Corollary 3.10. If we take $f=I$, the identity mapping in Theorem 3.9, then we get Corrolary 3.2.

Remark 3.11. If we take $\alpha=\frac{1-2 a-b}{1+c+b}$ and $f=I$ in Theorem 3.9, then we get Theorem 3.6 as a particular case.

\section{References}

[1] M. Berinde, V. Berinde, On a general class of multi-valued weakly Picard mappings, J. Math. Anal., 326 (2007), 772-782.

[2] R.K. Bose, R.N. Mukherjee, Common fixed points of some multi-valued mappings, Tamkang J. Math, 8, No. 2 (1977), 245-249.

[3] R.K. Bose, M.K. Roychowdhury, Fixed point theorems for some generalized contractivemulti-valued mappings and fuzzy mappings, Mathemtici Vesnik, 63, No. 1 (2011), 7-26.

[4] H. Covitz, S.B. Nadler Jr., Multivalued contraction mappings in generalized metric spaces, Israel J. Math., 8 (1970) 5-11. 
[5] S. Dhompongsa, H. Yingtaweesittikul, Fixed point for mulivalued mappings and the metric completeness, Fixed Point Theory and Applications (2009), 15, doi: 10.1155/2009/972395.

[6] Dragon Doric, Rade Lazovic, Some Suzuki-type fixed point theorems for generalized multivalued mappings and applications, Fixed point Theory and Applications, 40 (2011).

[7] T. Kamran, Multivalued $f$-weakly Picard mappings, Nonlinear Analysis, 67 (2007), 2289-2296.

[8] M. Kikkawa, T. Suzuki, Three fixed point theorems for generalized contractions with constants in complete metric space, Nonlinear Analysis, 69 (2008), 2942-2949.

[9] G. Mot, A. Petrusel, Fixed point theory for a new type of contracvtive multi-valued operators, Nonlinear Analysis, 70 (2009), 3371-3377.

[10] S.B. Nadler, Mutivalued contraction mappings, Pacific J. Math., 30 (1969), 475-488.

[11] S.L. Singh, S.N. Mishra, Remarks on recent fixed point theorems, Fixed Point Theory and Applications (2010), 18, doi: 1155/2010/452905.

[12] T. Suzuki, A generalized Banach contraction principle that charectreized metric completeness, Proceedings of the American Mathematical Society, 136 (2008), 1861-1869. 
